\title{
Distribuição sazonal de vetores da malária em Machadinho d'Oeste, Rondônia, Região Amazônica, Brasil
}

\author{
Seasonal distribution of malaria vectors \\ in Machadinho d'Oeste, Rondônia State, \\ Amazon Region, Brazil
}

Reinaldo Souza-Santos 1

\footnotetext{
1 Departamento de Endemias Samuel Pessoa, Escola Nacional de Saúde Pública, Fundação Oswaldo Cruz. Rua Leopoldo Bulhões 1480, Rio de Janeiro, $R J$ 21041-210, Brasil. rssantos@ensp.fiocruz.br
}

\begin{abstract}
The objective of this study was to describe the seasonal distribution of malaria vectors in Machadinho d'Oeste, Rondônia State, Brazil. The study was based on data from the Brazilian National Health Foundation (FUNASA) for the period from April 1993 to November 1994. A total of 97 field visits were conducted, and 93 intradomiciliary and 95 peridomiciliary collections were performed. The most frequently captured species in both the intra- and peridomiciles was Anopheles darlingi, followed by Anopheles triannulatus, among other species. Only An. darlingi was captured throughout the study period, and it was more abundant during the transition from the dry to the rainy season and vice versa. The elaboration of scientific studies based on secondary entomological data from FUNASA has been reinforced, since these results are comparable to those from the literature, taken from studies using primary data.
\end{abstract}

Key words Anopheles spp.; Insect Vectors; Malaria; Amazonian Ecosystem

Resumo O objetivo deste trabalho foi descrever a distribuição sazonal de vetores da malária em Machadinho d'Oeste, Estado de Rondônia. O estudo foi baseado em dados da Fundação Nacional de Saúde (FUNASA), referentes ao período de abril de 1993 a novembro de 1994. Foram efetuadas 97 visitas ao campo, correspondentes a 93 coletas no intra e 95 no peridomicílio. A espécie capturada em maior número, tanto no intra como no peridomicílio, foi Anopheles darlingi, seguido pela espécie Anopheles triannulatus, entre outras. Apenas An. darlingi foi capturada em todo o período estudado, sendo mais abundante nas estações de transição entre seca e chuva e vice-versa. A elaboração de trabalhos científicos baseados em dados secundários entomológicos oriundos da FUNASA é reforçada, uma vez que os resultados aqui encontrados equiparam-se aos existentes na literatura, procedentes de trabalhos com dados primários.

Palavras-chave Anopheles spp.; Insetos Vetores; Malária; Ecossistema Amazônico 


\section{Introdução}

Nas décadas de 80 e 90, observou-se um enorme aumento do número de casos de malária no sudoeste amazônico e, em especial, no Estado de Rondônia. Alguns municípios desse Estado apresentaram alarmantes níveis de Índice Parasitário Anual (IPA). Como exemplos, os Municípios de Jarú, Ariquemes e Machadinho d'Oeste registraram, em 1991, IPA nos valores de 203,7, 499,0 e 798,1, respectivamente (Escobar, 1994). Diante deste panorama, houve desenvolvimento de vários estudos visando ao conhecimento das espécies de mosquitos envolvidas na transmissão da malária na região (Deane et al., 1988; Klein \& Lima, 1990; Lourenço-de-Oliveira et al., 1989; Tadei et al., 1988; Tadei et al., 1998). Foram identificadas diferentes espécies de Anopheles como potencialmente vetoras de Plasmodium sp. em Rondônia, destacando-se o Anopheles darlingi e An. triannulatus. Em praticamente todos os trabalhos, foi evidenciada associação entre densidade populacional de anofelinos, variações climáticas sazonais e alterações ambientais de origem antropogênica.

O objetivo deste trabalho foi descrever a distribuição sazonal de vetores da malária, particularmente An. darlingi, no Município de Machadinho d'Oeste, Estado de Rondônia, visando à compreensão da situação entomológica local. O estudo baseou-se em informações secundárias disponibilizadas pela Fundação Nacional de Saúde (FUNASA). Desse modo, esse trabalho também visa contribuir para a discussão acerca do potencial de utilização de dados sobre vetores, coletados na rotina de programas de controle de endemias, na avaliação e no planejamento desses programas.

\section{Material e métodos}

O Município de Machadinho d'Oeste, Estado de Rondônia possui uma área geográfica de $11.715,80 \mathrm{~km}^{2} \mathrm{e}$, segundo o censo demográfico de 1996 (IBGE, 1996), conta com uma população de 28.849 habitantes e densidade demográfica de 1,59 habitantes $/ \mathrm{km}^{2}$ (Figura 1). A produção agropecuária constitui a principal atividade econômica do Município (http://www. rondonia.com/machadinho - consultado em 18/03/2002). A temperatura média anual no município é de $24^{\circ} \mathrm{C}$. A estação chuvosa é bem definida, entre os meses de dezembro e março, e a estação seca entre junho e agosto. As precipitações anuais são em torno de $2.000 \mathrm{~mm}$ e a umidade relativa do ar entre 80 e $85 \%$.
A presente análise foi baseada nas informações entomológicas coligidas pela FUNASA, em 21 localidades do Município de Machadinho d'Oeste, no período de abril de 1993 a novembro de 1994. De acordo com a FUNASA, cada localidade corresponde a um conjunto de lotes que possui uma ou mais residências, onde as coletas foram efetuadas no intra e peridomicílio. Tais informações foram categorizadas respeitando os seguintes critérios: (a) código da localidade onde os mosquitos foram coletados; (b) data da coleta; (c) local da coleta (intra e/ou peridomicílio); (d) total de mosquitos coletados, por espécie; (e) horário de início e (f) tempo de duração das coletas.

As coletas foram efetuadas com isca humana e contaram com a participação de oito agentes da FUNASA. Cada agente atuava, simultaneamente, como isca e capturador. Todas as capturas foram iniciadas às 18:00 e tiveram duração de três horas consecutivas. Embora existisse, no formulário da FUNASA, um campo para registro da data em que a localidade foi borrifada com inseticida pela última vez, ele não foi preenchido.

Com base nessas informações, foi criado um banco de dados para análise, utilizando-se o programa SPSS 9.0 (SPSS Incorporation, 1998). Apesar da existência de data completa da coleta, as informações foram agrupadas, inicialmente, segundo o mês e, posteriormente, em períodos de dois ou de três meses. Este procedimento teve o objetivo de melhor avaliar possíveis variações do número de mosquitos coletados, segundo estações climáticas (chuva e seca). Os meses de 1993 foram agrupados do seguinte modo: abril, maio e junho - período 1 (transição chuvaseca); julho e agosto - período 2 (seca); setembro, outubro e novembro - período 3 (transição seca-chuva); não existindo informações para os meses de janeiro a março e dezembro. Em virtude da inexistência de informações para alguns meses de 1994, os demais foram agrupados do seguinte modo: março - período 4 (chuva); maio e junho - período 5 (transição chuva-seca); julho e agosto - período 6 (seca); setembro, outubro e novembro - período 7 (transição seca-chuva). A ausência dessas informações deveu-se à perda de dados por parte do serviço de saúde (como no caso de abril de 1994) ou à impossibilidade de desenvolvimento dos trabalhos de campo (em função de aspectos logísticos e meteorológicos) pelos agentes da FUNASA.

Para a análise da variação de $A n$. darlingi em relação aos períodos correspondentes às estações de seca e de chuva, calculou-se a média de mosquitos coletados em cada período, para o intra e peridomicílio, separadamente 
(total de An. darlingi coletado no período no intra ou peridomicílio/média mensal de coletas). Com o objetivo de reduzir a influência da variação do número de coletas em cada período, utilizou-se a média mensal de coletas efetuadas ao longo dos dois anos, como ponderador (total de coletas no intra e peridomicílio, separadamente/total de meses trabalhados).

\section{Resultados e discussão}

Durante o período do estudo, foram realizados 97 trabalhos de campo, que totalizaram $188 \mathrm{co-}$ letas (93 no intra e 95 no peridomicílio) distribuídas em 21 diferentes localidades, (média de 4,6 coletas por localidade). Das 93 coletas realizadas no intradomicílio, 92 (98,9\%) contaram com a participação de um único agente, e das 95 realizadas no peridomicílio, 68 (71,6\%) foram efetuadas por dois agentes.

O serviço de vigilância entomológica tem como objetivo, como o próprio nome indica, acompanhar as variações nas populações de vetores no município, independente da existência de áreas com maior incidência da doença. Contudo, ao se considerar o total de localidades registradas pela FUNASA para o Município de Machadinho d'Oeste (163 localidades), verifica-se que o levantamento entomológico foi efetuado em apenas 12,9\% (21 localidades). Isso se deveu ao direcionamento dos levantamentos entomológicos para as localidades onde, na época (entre 1993 e 1994), registraramse maiores incidências de malária e que correspondiam a assentamentos agropecuários recentemente instalados em diferentes regiões do município. Assim sendo, as localidades restantes permaneceram, naquele período, sem vigilância entomológica, realizando exclusivamente o registro de casos de malária.

Como observado em outros estudos desenvolvidos na região amazônica, inclusive em Rondônia, An. darlingi foi a espécie capturada em maior número, tanto no intra quanto no peridomicílio (Deane et al., 1988; Klein \& Lima, 1990; Lourenço-de-Oliveira et al., 1989; Oliveira-Ferreira et al., 1990; Tadei et al., 1988; Tadei \& Thatcher, 2000). An. triannulatus foi a segunda espécie capturada em maior número, também no intra e no peridomicílio, seguida por $A n$. rangeli, An. braziliensis e An. nuneztovari (Tabela 1). An. rangeli, An. braziliensis e An. nuneztovari não foram capturadas em todos os períodos, concentrando-se, sobretudo, naqueles correspondentes às estações de transição (Tabela 1).

Segundo os estudos de Deane et al. (1988), Oliveira-Ferreira et al. (1990), Tadei et al. (1998)
Figura 1

Município de Machadinho d'Oeste, Estado de Rondônia, Brasil.

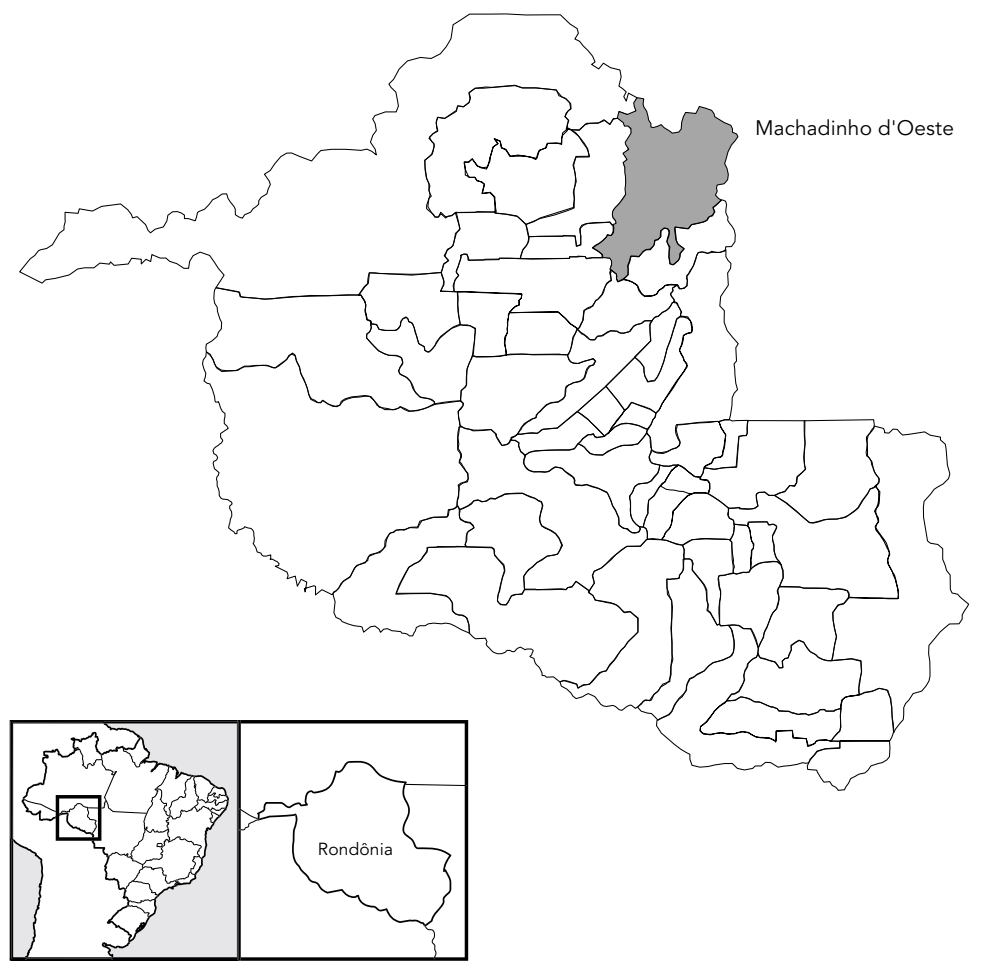

e Tadei \& Thatcher (2000), dentre outros, $A n$. darlingi é a principal espécie transmissora da malária na região. Apesar disso, esses autores comentam que An. triannulatus, An. braziliensis e An. nuneztovari já foram encontradas infectadas com Plasmodium falciparum e/ou $P$. vivax, também estando envolvidas, ainda que em menor escala, na transmissão da malária.

Ao observar as variações do número de espécimes, de todas as espécies capturadas, ao longo do período do estudo e estações climáticas, nota-se uma relação positiva entre os períodos de transição (chuva/seca e seca/chuva) e o aumento do número de mosquitos capturados. Somente a espécie An. darlingi foi capturada em todos os sete períodos do estudo, tanto no intra como no peridomicílio. Todas as outras espécies foram capturadas principalmente nos períodos de transição, períodos 1 , 3 , 5 e 7 (Tabela 1). Vale ressaltar que no período 4 (chuva) foram efetuadas apenas duas coletas. A redução ou a inexistência de coletas nos períodos de chuva devem-se principalmente às dificuldades de acesso às localida- 
Freqüência de fêmeas de mosquitos coletadas em Machadinho d’Oeste, Rondônia, Brasil, segundo espécie e estação do ano (1993-1994).

\begin{tabular}{|c|c|c|c|c|c|c|c|c|c|c|c|c|}
\hline \multirow{2}{*}{ Períodos ${ }^{\star \star}$} & \multicolumn{2}{|c|}{ An. darlingi } & \multicolumn{2}{|c|}{ An. triannulatus } & \multicolumn{2}{|c|}{ An. rangeli } & \multicolumn{2}{|c|}{ An. braziliensis } & \multicolumn{2}{|c|}{ An. nuneztovari } & \multicolumn{2}{|c|}{ Anopheles spp.* } \\
\hline & Peri & Intra & Peri & Intra & Peri & Intra & Peri & Intra & Peri & Intra & Peri & Intra \\
\hline \multirow[t]{2}{*}{1 Chuva/seca } & 2.270 & 591 & 24 & 3 & 10 & 2 & 16 & 3 & 6 & 2 & 25 & 1 \\
\hline & $(36,81)$ & $(37,74)$ & $(12,31)$ & $(11,11)$ & $(19,23)$ & $(18,18)$ & $(55,17)$ & $(75,00)$ & $(27,27)$ & $(40,00)$ & $(69,44)$ & $(33,33)$ \\
\hline \multirow[t]{2}{*}{2 Seca } & 104 & 25 & 7 & 0 & 1 & 0 & 0 & 0 & 0 & 0 & 2 & 0 \\
\hline & $(1,69)$ & $(1,60)$ & $(3,59)$ & $(0,00)$ & $(1,92)$ & $(0,00)$ & $(0,00)$ & $(0,00)$ & $(0,00)$ & $(0,00)$ & $(5,56)$ & $(0,00)$ \\
\hline \multirow[t]{2}{*}{$3 \mathrm{Seca} / \mathrm{chuva}$} & 684 & 156 & 37 & 4 & 2 & 0 & 0 & 0 & 1 & 3 & 5 & 0 \\
\hline & $(11,09)$ & $(9,96)$ & $(18,97)$ & $(14,81)$ & $(3,85)$ & $(0,00)$ & $(0,00)$ & $(0,00)$ & $(4,55)$ & $(60,00)$ & $(13,89)$ & $(0,00)$ \\
\hline \multirow[t]{2}{*}{4 Chuva } & 200 & 23 & 1 & 0 & 0 & 0 & 0 & 0 & 0 & 0 & 1 & 0 \\
\hline & $(3,24)$ & $(1,47)$ & $(0,51)$ & $(0,00)$ & $(0,00)$ & $(0,00)$ & $(0,00)$ & $(0,00)$ & $(0,00)$ & $(0,00)$ & $(2,78)$ & $(0,00)$ \\
\hline \multirow[t]{2}{*}{5 Chuva/seca } & 1.869 & 538 & 24 & 9 & 0 & 0 & 9 & 1 & 4 & 0 & 2 & 2 \\
\hline & $(30,31)$ & $(34,36)$ & $(12,31)$ & $(33,33)$ & $(0,00)$ & $(0,00)$ & $(31,03)$ & (25) & $(18,18)$ & $(0,00)$ & $(5,56)$ & $(66,67)$ \\
\hline \multirow[t]{2}{*}{6 Seca } & 26 & 5 & 1 & 0 & 3 & 0 & 0 & 0 & 2 & 0 & 0 & 0 \\
\hline & $(0,42)$ & $(0,32)$ & $(0,51)$ & $(0,00)$ & $(5,77)$ & $(0,00)$ & $(0,00)$ & $(0,00)$ & $(9,09)$ & $(0,00)$ & $(0,00)$ & $(0,00)$ \\
\hline \multirow[t]{2}{*}{$7 \mathrm{Seca} / \mathrm{chuva}$} & 1.014 & 228 & 101 & 11 & 36 & 9 & 4 & 0 & 9 & 0 & 1 & 0 \\
\hline & $(16,44)$ & $(14,56)$ & $(51,79)$ & $(40,74)$ & $(69,23)$ & $(81,82)$ & $(13,79)$ & $(0,00)$ & $(40,91)$ & $(0,00)$ & $(2,78)$ & $(0,00)$ \\
\hline Total & 6.167 & 1.566 & 195 & 27 & 52 & 11 & 29 & 4 & 22 & 5 & 36 & 3 \\
\hline$N=8.117$ & $(75,98)$ & $(19,29)$ & $(2,40)$ & $(0,33)$ & $(0,64)$ & $(0,13)$ & $(0,35)$ & $(0,04)$ & $(0,27)$ & $(0,06)$ & $(0,44)$ & $(0,03)$ \\
\hline
\end{tabular}

* Outros: An. albitarsis, An. evansae, An. oswaldoi e An. cruzii (An. cruzii foi coletado apenas no peridomicílio).

** Períodos: 1 - abril, maio e junho (transição chuva/seca); 2 - julho e agosto (seca); 3 - setembro, outubro e novembro (transição seca/chuva);

4 - março (chuva); 5 - maio e junho (transição chuva/seca); 6 - julho e agosto (seca); 7 - setembro, outubro e novembro (transição seca/chuva).

des e à redução do número de coleta de espécimes adultos.

A média de coletas mensais no intradomicílio foi 5,81 e, no peridomicílio, 5,94. Ao observar as médias de captura de An. darlingi, no intra e peridomicílio, ponderadas pelas médias mensais de coletas, verifica-se que as variações seguem o mesmo padrão (Figura 2). A variação temporal aqui encontrada é semelhante à observada por outros autores, existindo uma relação positiva entre o aumento do número de espécimes capturados e o início e fim dos períodos das chuvas, ou seja, nas estações de transição (Klein \& Lima, 1990; Tadei et al., 1998).

O gênero Anopheles inclui diversas espécies de interesse epidemiológico. Os resultados aqui apresentados apontam para An. darlingi como a espécie mais freqüentemente capturada no peridomicílio, mormente durante as estações de transição.

Para a melhor compreensão da epidemiologia da malária, pesquisas que mostrem as espécies potencialmente vetoras existentes em uma determinada região, bem como a freqüên- cia de cada uma e sua variação ao longo do ano, são de extrema relevância. A variação no número de casos de malária está, além de outros fatores, relacionada às flutuações do número de seus vetores. Ainda que haja, ao longo do ano, picos no número de espécimes capturados, os resultados aqui apresentados revelam a presença de An. darlingi em todas as estações, tanto no intra como no peridomicílio. Esses resultados deixam claro que as ações de controle não podem ficar restritas aos períodos com maiores densidades do vetor, devendo ser efetuadas durante todo o ano.

O percentual de localidades cobertas pelo serviço de vigilância entomológica $(12,9 \%)$ também evidencia a necessidade de novo direcionamento espacial nas atividades de controle. Apesar da impossibilidade de extrapolação dos resultados para áreas com características ambientais diferentes, tais como áreas urbanas e silvestres, o conhecimento sobre os vetores da malária em áreas onde foi verificado aumento na incidência de malária é de grande importância. Acrescente-se que áreas potencialmente en- 


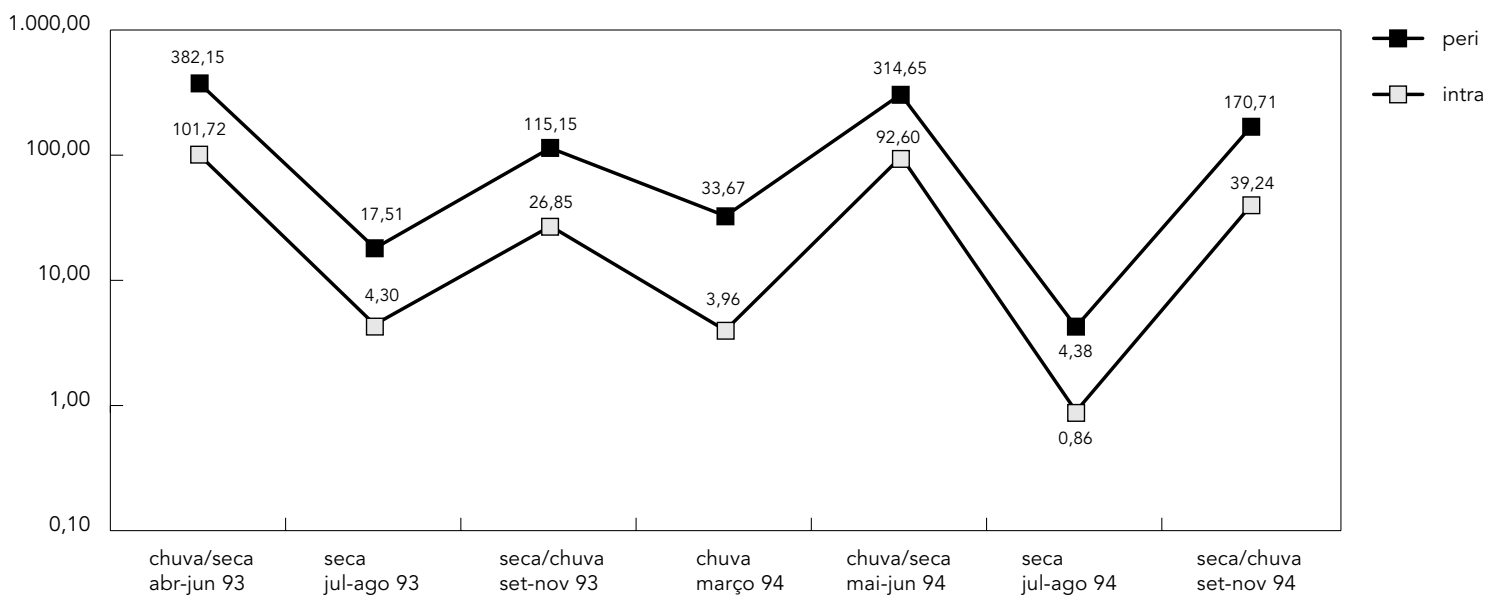

dêmicas para malária, ou onde possam existir criadouros de anofelinos, devem ser submetidas à vigilância.

Em razão das falhas existentes nos sistemas de vigilância entomológica e programas de controle, análises de fauna de vetores com base em dados secundários tendem a ser questionadas por causa da baixa confiabilidade dos dados. No entanto, as informações não podem ser ignoradas. Deve ser estimulado, isto sim, seu uso crítico, objetivando o aperfeiçoamento dos sistemas e programas de informação e das atividades de vigilância entomológica (FlemingMoran, 1992; Souza-Santos, 1999; Souza-Santos \& Carvalho, 2000).
Cumpre destacar que os resultados oriundos da análise das informações sobre os vetores da malária, coletados pela FUNASA em Machadinho d'Oeste, estão de acordo, em linhas gerais, com a literatura sobre estudos entomológicos de campo na região, o que aponta para a importância de serem desenvolvidas estratégias que propiciem a análise de bases de dados secundários. Estratégias geradas pelos serviços de saúde com intuito de melhorar o entendimento da epidemiologia de endemias em uma determinada região.

\section{Agradecimentos}

O autor agradece à Fundação Nacional de Saúde de Machadinho d'Oeste o fornecimento das informações e aos professores Carlos E. A. Coimbra Jr. (Escola Nacional de Saúde Pública, Fundação Oswaldo Cruz) e Ana Lúcia Escobar (Universidade Federal de Rondônia) as críticas. 


\section{Referências}

DEANE, L. M.; RIBEIRO, C. D.; LOURENÇO-DE-OLIVEIRA, R.; OLIVEIRA-FERREIRA, J. \& GUIMARÃES, A. E., 1988. Study on the natural history of malaria in areas of the Rondonia State, Brazil, and problems related to its control. Revista do Instituto de Medicina Tropical de São Paulo, 30:153-156.

ESCOBAR, A. L., 1994. Malária no Sudoeste da Amazônia: Uma Meta-análise. Dissertação de Mestrado, Rio de Janeiro: Escola Nacional de Saúde Pública, Fundação Oswaldo Cruz.

FLEMING-MORAN, M., 1992. The initial success of the Chagas' Disease Control Program: Factors contributing to Triatomine infestation. Cadernos de Saúde Pública, 8:391-403.

IBGE (Fundação Instituto Brasileiro de Geografia e Estatística), 1996. Contagem Populacional 1996. 27 Maio $2002<$ http:www.ecof.org.br/projetos/ machadi $2 />$.

KLEIN, T. A. \& LIMA, J. B. P., 1990. Seasonal distribution and biting patterns of Anopheles mosquitoes in Costa Marques, Rondonia, Brazil. Journal of the American Mosquito Control Association, 6:700707.

LOURENÇO-DE-OLIVEIRRA, R.; GUIMARÃES, A. E. G.; ARLÉ, M.; SILVA, T. F.; CASTRO, M. G.; MOTTA, M. A. \& DEANE, L. M., 1989. Anopheline species, some of their habits and relation to malaria in endemic areas of Rondônia State, Amazon Region of Brazil. Memórias do Instituto Oswaldo Cruz, 84:501-514.
OLIVEIRA-FERREIRA, J.; LOURENÇO-DE-OLIVEIRA, R.; TEVA, A.; DEANE, L. M. \& DANIEL-RIBEIRO, C. T., 1990. Natural malaria infections in anophelines in Rondônia State, Brazilian Amazon. American Journal of Tropical Medicine and Hygiene, 43:6-10.

SOUZA-SANTOS, R., 1999. Fatores associados à ocorrência de formas imaturas de Aedes aegypti na Ilha do Governador, Rio de Janeiro, Brasil. Revista da Sociedade Brasileira de Medicina Tropical, 32:373382.

SOUZA-SANTOS, R. \& CARVALHO, M. S., 2000. Análise da distribuição espacial de larvas de Aedes aegypti na Ilha do Governador, Rio de Janeiro, Brasil. Cadernos de Saúde Pública, 16:31-42.

SPSS INCORPORATION, 1998. SPSS for Windows. Statistical Package for the Social Sciences. Release 9.0. Chicago: SPSS Inc.

TADEI, W. P.; SANTOS, J. M. M.; COSTA, W. L. S. \& SCARPASSA, V. M., 1988. Biologia de anofelinos amazônicos. XVII. Ocorrência de espécies de Anopheles, dinâmica da transmissão e controle da malária na zona urbana de Ariquemes (Rondônia). Revista do Instituto de Medicina Tropical de São Paulo, 30:221-251.

TADEI, W. P. \& THATCHER, B. D., 2000. Malaria vectors in the Brazilian Amazon: Anopheles of the subgenus Nyssorhynchus. Revista do Instituto de Medicina Tropical de São Paulo, 42:87-94.

TADEI, W. P.; THATCHER, B. D.; SANTOS, J. M. M.; SCARPASSA, V. M.; RODRIGUES, I. B. \& RAFAEL, M. S., 1998. Ecologic observations on anopheline vectors of malaria in the Brazilian Amazon. American Journal of Tropical Medicine and Hygiene, 59:325-335.

Recebido em 1 de abril de 2002

Versão final reapresentada em 10 de junho de 2002

Aprovado em 17 de julho de 2002 